\title{
Daughter cysts in a cyst of the liver: hepatic echinococcosis
}

\author{
Byoung Woon Kwon, Seong Jun Park, Jae Hwan Kong, and Il Han Song
}

Department of Internal Medicine, Dankook University College of Medicine, Cheonan, Korea

Received: March 4, 2015

Revised : March 5, 2015

Accepted: March 5, 2015

\section{Correspondence to}

Il Han Song, M.D.

Tel: +82-41-550-3924

Fax: +82-41-556-3256

E-mail: ihsong21@dankook.ac.kr
A 58-year-old male from North-East China who was an immigrant worker for 4 years, presented with a cystic mass of the liver that was incidentally detected on abdominal ultrasound. Blood liver battery showed no abnormal findings and stool helminth was negative. Serologic markers for hepatitis A, B, and C virus were all negative and blood levels of tumor markers including $\alpha$-fetoprotein, carcinoembryonic antigen, and carbohydrate antigen 19-9 were within normal limits. Computed tomography (Fig. 1A, coronal view) and magnetic resonance imaging (Fig. 1B, axial view) of the liver revealed an approximately $9 \mathrm{~cm}$-sized non-enhancing exophytic cyst with several daughter cysts, a radiologic finding typical of cystic echinococcosis of the liver, on the left lobe lateral segment of the liver. Surgical resection was performed for complete removal. Grossly, the mass showed a $9.5 \times 9.5 \times 8.5 \mathrm{~cm}$-sized yellowish unilocular cyst containing several variable-sized cysts (Fig. 2A). Microscopically, a brood of capsules containing degenerated protoscolices of Echinococcus granulosus (arrows) were scattered on the laminated cystic walls (Fig. 2B). After uncomplicated postoperative recovery, the patient was discharged with albendazole $400 \mathrm{mg}$ twice daily for 4 weeks, with encouragement to consume fatrich meal diet. Hepatic echinococcosis, also called hydatid disease of the liver, is a representative zoonosis that contributes to major health public problem in several countries. It usually occurs as a result of infection by larval stage of species E. granulosus, genus Echinococcus, via
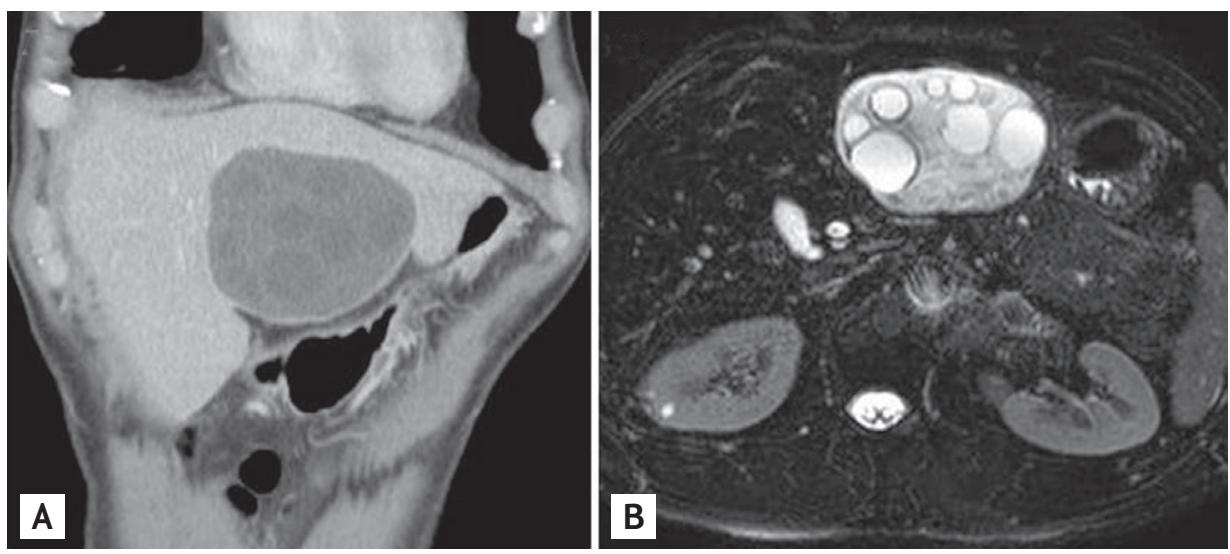

Figure 1. Liver computed tomography (A, coronal view) and magnetic resonance imaging (B, axial view) revealed a huge non-enhancing exophytic cyst with several daughter cysts on lateral segment of the left lobe of the liver. 

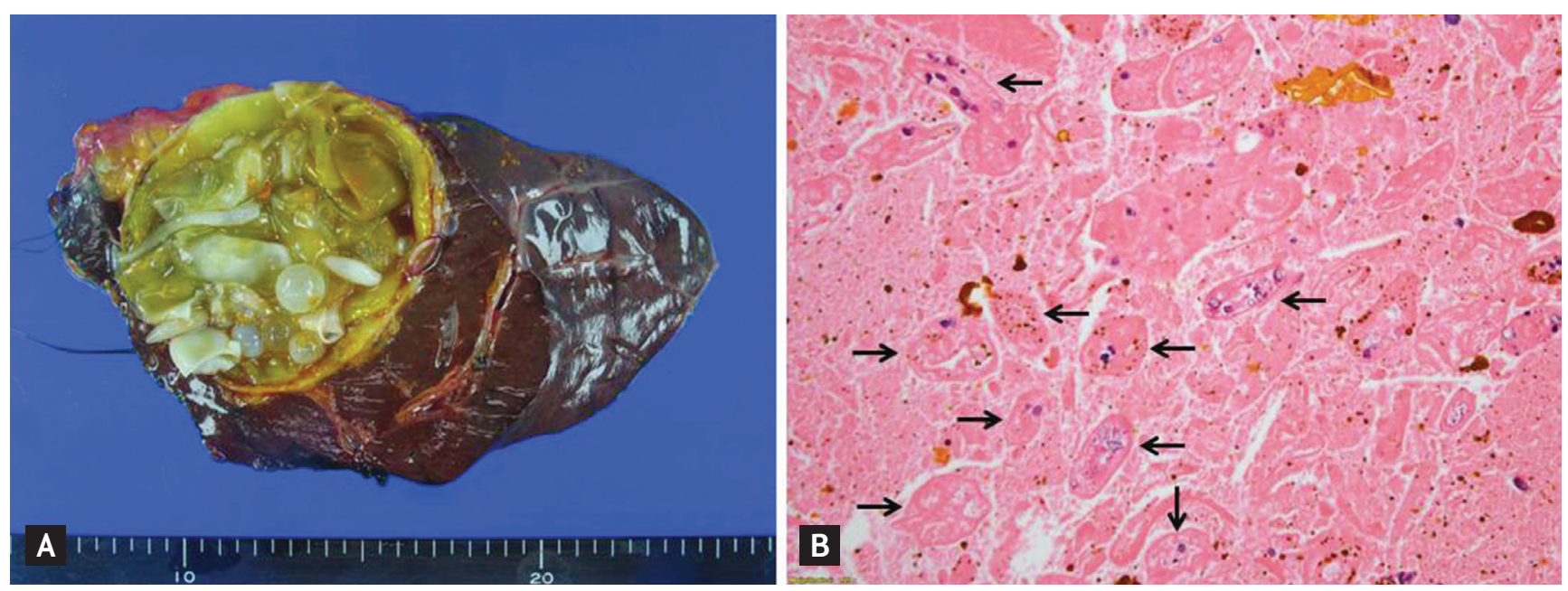

Figure 2. (A) Surgically resected mass showed a $9.5 \times 9.5 \times 8.5 \mathrm{~cm}$-sized yellowish unilocular cyst containing variable-sized several cysts. (B) Microscopically, a brood of capsules containing degenerated protoscolices of Echinococcus granulosus (arrows) were scattered on the laminated cystic walls $(\mathrm{H} \& \mathrm{E}, \times 200)$.

the ingestion of food and water fecally contaminated with eggs from the definitive hosts such as dogs and wolves, indicating that humans are an accidental intermediate host of the infection. Surgical resection followed by antihelminthic prophylaxis with albendazole remains the standard of care rather than percutaneous-based thera- peutic approach.

\section{Conflict of interest}

No potential conflict of interest relevant to this article was reported. 